\title{
The safety of performing breast reconstruction during the COVID-19 pandemic
}

\author{
Heather R. Faulkner ${ }^{1,2}\left(\right.$ D Suzanne B. Coopey ${ }^{3,4} \cdot$ Eric C. Liao $^{2} \cdot$ Michelle Specht $^{4} \cdot$ Barbara L. Smith $^{4} \cdot$ Amy S. Colwell $^{2}$
}

Received: 10 May 2021 / Accepted: 7 October 2021 / Published online: 15 October 2021

(c) The Author(s), under exclusive licence to The Japanese Breast Cancer Society 2021

\begin{abstract}
Purpose Elective operations including surgeries for breast cancer were significantly reduced during the height of the surge of COVID-19 cases in Massachusetts. The safety of performing breast reconstruction during the pandemic was unknown. This study aims to review the safety of performing mastectomy with immediate breast reconstruction during the first COVID-19 surge in Massachusetts.

Methods A retrospective chart review of patients who underwent mastectomy with immediate breast reconstruction by Massachusetts General Hospital breast and plastic surgeons immediately preceding and during the COVID-19 pandemic was performed.

Results Thirty patients (34 breasts) underwent mastectomies with immediate breast reconstruction during the COVID-19 restriction period in Massachusetts. Most reconstructions were unilateral. All reconstructions were performed with implants or expanders, and no autologous reconstructions were performed. Two patients ( 2 breasts) had operative complications. The complication rate during the pandemic was similar to the complication rate pre-pandemic. No patients or surgeons experienced symptoms or positive COVID-19 tests. Over $90 \%$ of patients were discharged the same day.

Conclusion Prosthetic breast reconstruction was able to be performed safely during the height of the COVID-19 pandemic surge in Massachusetts. Strict screening protocols, proper use of personal protective equipment, and same-day discharge when possible are essential for patient and surgeon safety during the pandemic.
\end{abstract}

Keywords Breast reconstruction · Breast cancer · Mastectomy $\cdot$ COVID-19 $\cdot$ Pandemic $\cdot$ Patient safety $\cdot$ Outcomes

\section{Introduction}

The COVID-19 pandemic is one of the most historic medical crises of the modern day. During the height of the COVID-19 pandemic, the governor of the state of Massachusetts declared a state of emergency on March 10, 2020 [1]. Restrictions on non-essential surgery (including breast

Heather R. Faulkner

hrfaulkner@alumni.harvard.edu

1 Present Address: Emory University Division of Plastic Surgery, Atlanta, GA, USA

2 Division of Plastic and Reconstructive Surgery, Massachusetts General Hospital/Harvard Medical School, Boston, MA, USA

3 Present Address: Allegheny Health Network, Division of Surgical Oncology, Wexford, PA, USA

4 Division of Surgical Oncology, Massachusetts General Hospital/Harvard Medical School, Boston, MA, USA cancer surgery) were enacted by the Massachusetts Department of Public Health on March 15, 2020 [1]. These restrictions continued until May 11, 2020, when special approval was granted for some oncologic procedures. Restrictions on surgery were lifted on June 24, 2020.

Priority for breast cancer surgery during the COVID-19 restriction period followed recommendations outlined by the COVID-19 Pandemic Breast Cancer Consortium and were decided in a multidisciplinary manner with input from medical oncology, surgical oncology, and radiation oncology $[2,3]$. In general, neoadjuvant chemotherapy, targeted HER2 therapy, or hormonal therapy was considered for all newly diagnosed patients and surgery was delayed if possible [2]. Clinical scenarios in which breast cancer surgery was permitted included: patients completing or disease progressing on neoadjuvant chemotherapy, patients in which pathologic staging information from surgery would impact systemic therapy recommendations (for example: clinical T1N0 HER2+ or triple-negative cancers), and some patients 
with local recurrences. Patients eligible for breast conservation were discouraged from mastectomy. Contralateral prophylactic mastectomies were deferred.

During the restriction period for non-essential surgery, there were multidisciplinary discussions on local, national, and international levels regarding whether breast reconstruction was considered essential. On March 24, 2020, the American Society of Plastic Surgeons issued a statement on breast reconstruction during the COVID-19 pandemic [4]. The statement recommended to evaluate immediate prosthetic reconstruction on a case-by-case basis, and to defer autologous reconstruction. Our plastic surgery faculty unanimously agreed to continue immediate prosthetic breast reconstructions, a decision which was supported by hospital administration. At the onset of the pandemic, there were concerns regarding an increased incidence of venous thromboembolic events in patients with COVID-19, and we did not have the ability to perform routine testing of patients preoperatively. This study examines the outcomes of patients who underwent prosthetic breast reconstruction during the restriction period in Massachusetts during the first surge of the COVID-19 pandemic.

\section{Patients and methods}

After obtaining approval from the Massachusetts General Hospital/Mass General Brigham Healthcare Institutional Review Board, patients who underwent mastectomy with immediate breast reconstruction by one of our faculty members during the COVID-19 pandemic first surge were identified. Faculty members who perform predominantly delayed prosthetic breast reconstructions were excluded. The review commenced with the first date operations were postponed as a result of the pandemic (March 15, 2020) and ended on May 31, 2020. This end date was chosen due to the organizational start of routine pre-operative COVID-19 testing for all elective operations on June 1, 2020. The COVID-19 restriction period was compared to the 3 preceding months. All operations during the COVID-19 restriction period were performed with enhanced respiratory isolation protocols.

The following data were culled: patient demographics, medical history, COVID-19 testing results, details of surgery, pathology results, complications, and neoadjuvant/ adjuvant treatment with chemotherapy and/or radiation. Study data were collected and managed using REDCap electronic data capture tools hosted at Partners Healthcare. REDCap (Research Electronic Data Capture) is a secure, web-based application designed to support data capture for research studies, providing (1) an intuitive interface for validated data entry; (2) audit trails for tracking data manipulation and export procedures; (3) automated export procedures for seamless data downloads to common statistical packages; and (4) procedures for importing data from external sources. Data were analyzed using Stata/IC version 15.1 (College Station, Texas). Two-tailed t-tests (and Fisher exact tests where applicable) were used to compare categorical variables. Linear regression was used to compare continuous variables. Two-sample tests of proportions were used to compare outcomes prior to COVID-19 to outcomes during the COVID-19 restriction period. Alpha was set at the standard of 0.05 .

\section{Results}

During the 3 months prior to COVID-19 (pre-C19), 47 patients underwent 77 mastectomies with immediate reconstruction (17 unilateral (36.2\%), 30 bilateral (63.8\%)). Mastectomies were performed in the following formats: nipplesparing 60 breasts (77.9\%), skin-sparing 13 breasts (16.9\%), areola-sparing 4 breasts (5.2\%). Forty breasts (51.9\%) were reconstructed in the prepectoral plane (37 direct to implant and 3 expanders) and 37 breasts (48.1\%) were reconstructed in the subpectoral plane (27 direct to implant and 10 expanders). Sixty-four breasts (83.1\%) were direct to implant reconstruction and 13 breasts (16.9\%) were reconstructed with expanders. Reconstruction details are listed in Table 1. Complications during pre-C19 requiring reoperation occurred in 3 breasts (3.9\%). Five patients ( 5 breasts, $6.5 \%$ ) had minor complications. Length of stay was 0 nights (same-day discharge) in 2 patients (4.3\%), 1 night in 44 patients (93.6\%), and 2 nights in 1 patient (2.1\%). Details of complications are listed in Table 2.

Table 1 Breast reconstruction during Pre-C19 and R-C19

\begin{tabular}{|c|c|c|c|c|c|}
\hline \multirow[t]{2}{*}{ Variable } & \multicolumn{2}{|c|}{$\begin{array}{l}\text { Pre-C19 } \\
\text { (47 patients, } 77 \\
\text { breasts) }\end{array}$} & \multicolumn{2}{|c|}{$\begin{array}{l}\text { R-C19 } \\
\text { (30 patients, } 34 \\
\text { breasts) }\end{array}$} & \multirow[t]{2}{*}{$p$} \\
\hline & $N$ & Percent & $N$ & Percent & \\
\hline \multicolumn{6}{|l|}{ Side } \\
\hline Unilateral & 17 & 36.2 & 26 & 86.7 & \multirow[t]{2}{*}{$<0.001$} \\
\hline Bilateral & 30 & 63.8 & 4 & 13.3 & \\
\hline \multicolumn{6}{|l|}{ Mastectomy type } \\
\hline Nipple-sparing & 60 & 77.9 & 20 & 58.9 & \multirow[t]{3}{*}{0.0387} \\
\hline Skin-sparing & 13 & 16.9 & 9 & 26.5 & \\
\hline Areola-sparing & 4 & 5.2 & 5 & 14.7 & \\
\hline \multicolumn{6}{|l|}{ Plane } \\
\hline Prepectoral & 40 & 51.9 & 24 & 70.6 & \multirow[t]{2}{*}{0.0669} \\
\hline Subpectoral & 37 & 48.1 & 10 & 29.4 & \\
\hline \multicolumn{6}{|l|}{ Method } \\
\hline Direct to Implant & 64 & 83.1 & 24 & 70.6 & \multirow[t]{2}{*}{0.1333} \\
\hline Expander & 13 & 16.9 & 10 & 29.4 & \\
\hline
\end{tabular}


Table 2 Complications during Pre-C19 and R-C19

\begin{tabular}{|c|c|c|c|c|c|}
\hline \multirow[t]{2}{*}{ Variable } & \multicolumn{2}{|c|}{ Pre-C19 (77 breasts) } & \multicolumn{2}{|c|}{ R-C19 (34 breasts) } & \multirow[t]{2}{*}{$p$} \\
\hline & $N$ (breasts) & $\overline{\text { Percent }}$ & $N$ (breasts) & $\overline{\text { Percent }}$ & \\
\hline Operative complications & 3 & 3.9 & 2 & 5.9 & 0.6419 \\
\hline Non-operative complications & 5 & 6.5 & 3 & 8.8 & 0.6617 \\
\hline Infection requiring surgery & $1^{\mathrm{a}}$ & 1.3 & $1^{\mathrm{c}}$ & 2.9 & \\
\hline Infection-non-operative & 3 & 3.9 & 1 & 2.9 & \\
\hline Necrosis requiring surgery & $2^{\mathrm{b}}$ & 2.6 & $1^{\mathrm{c}}$ & 2.9 & \\
\hline Necrosis-non-operative & 2 & 2.6 & 2 & 5.9 & \\
\hline Implant/expander removal & $1^{\mathrm{a}}$ & 1.3 & $1^{\mathrm{c}}$ & 2.9 & \\
\hline Implant/expander exposure & 0 & 0 & $1^{\mathrm{c}}$ & 2.9 & \\
\hline Hematoma & 0 & 0 & 1 & 2.9 & \\
\hline Seroma & $1^{\mathrm{a}}$ & 1.3 & 0 & 0 & \\
\hline Capsular contracture & 0 & 0 & 0 & 0 & \\
\hline Implant malposition & 0 & 0 & 0 & 0 & \\
\hline Venous thromboembolism & 0 & 0 & 0 & 0 & \\
\hline Mortality & 0 & 0 & 0 & 0 & \\
\hline
\end{tabular}

${ }^{a}$ One patient experienced mastectomy flap necrosis with infected seroma requiring expander removal

${ }^{\mathrm{b}}$ One patient had mastectomy flap necrosis bilaterally which required operative debridement for salvage

${ }^{c}$ One patient had mastectomy flap necrosis with infection and implant exposure requiring implant removal
During the COVID-19 restriction period (R-C19), 30 patients underwent 34 mastectomies with immediate reconstruction (26 unilateral (86.7\%), 4 bilateral (13.3\%)). Although there were restrictions on bilateral cases, 4 patients underwent bilateral operations during R-C19. One bilateral case was done on March 17, 2020, before the guidelines for breast cancer surgery during COVID-19 were enacted. One bilateral case was performed with special permission due to genetic mutation in NF1 and high risk for contralateral breast cancer [5]. The other two cases were performed in late May 2020 in a Mass General Brigham community hospital with permission granted due to resource availability in addition to patient factors (both with a current diagnosis of breast cancer and considered high risk for contralateral breast cancer due to young age and/or family history, but without genetic mutations).

Eleven patients were initially planning to have bilateral mastectomies (all had unilateral cancer and wished to have a contralateral prophylactic mastectomy for risk reduction), but had unilateral surgery as a result of restrictions imposed during R-C19. Since restrictions were lifted, three of the eleven patients have returned to have their contralateral prophylactic mastectomy with prosthetic reconstruction (mean 2.5 months from their initial operation). No post-operative complications occurred within 30 days for this cohort of patients. The remaining 8 patients are being followed with mammography.

Two patients were tested for COVID pre-operatively. Both were tested in late May 2020 due to the early commencement of routine pre-operative testing within the Division of Plastic Surgery, and were negative. Eight patients were tested for COVID post-operatively (either for symptoms or for additional planned operations), and all were negative. No patients underwent testing for COVID-19 antibodies. Surgery during R-C19 was performed by 4 breast surgeons and 5 plastic surgeons.

Patients undergoing breast reconstruction during R-C19 had the following characteristics: mean BMI 27.0 (range 20-41.1), mean age at surgery 48.4 (range 26.5-75). Diagnoses included: 3 breasts with DCIS only, 4 breasts with invasive cancer only, 23 breasts with both DCIS and invasive cancer, and 4 (prophylactic) breasts with benign pathology. Three patients had prior radiation and 8 patients had postmastectomy radiation. Twelve patients completed neoadjuvant chemotherapy and 9 patients underwent adjuvant chemotherapy. Comorbidities, medical history, surgical history, family history, social history, and cancer therapies of the $\mathrm{R}-\mathrm{C} 19$ cohort are listed in Table 3.

Mastectomies during R-C19 were performed in the following formats: nipple-sparing 20 breasts (58.8\%), skinsparing 9 breasts (26.4\%), areola-sparing 5 breasts (14.7\%). Reconstructions were performed exclusively with implants or expanders during R-C19. Twenty-four breasts (70.6\%) were direct to implant reconstruction and 10 breasts (29.4\%) were reconstructed with expanders. Twenty-four breasts (70.6\%) were reconstructed in the prepectoral plane (20 direct to implant, 4 expanders), 10 breasts (29.4\%) were reconstructed in the subpectoral plane (4 direct to implant, 6 expanders). Adjunctive materials were used in all reconstructions: 20 reconstructions (58.8\%) used acellular dermal matrix only, 12 reconstructions (35.3\%) used acellular dermal matrix and vicryl mesh, and 2 reconstructions (5.9\%) 
Table 3 Medical history, surgical history, cancer treatment-R-C19 patients

\begin{tabular}{lll}
\hline Variable & $\mathrm{N}$ (patients) & Percent \\
\hline Hypertension & 8 & 26.7 \\
Diabetes & 1 & 3.3 \\
Smoking & & \\
Never & 23 & 76.7 \\
Current & 1 & 3.3 \\
Former & 6 & 20.0 \\
Alcohol & & \\
None & 9 & 30.0 \\
1-2 per week/social & 8 & 26.7 \\
3-10 per week & 13 & 43.3 \\
Genetic testing & & \\
Negative & 23 & 76.7 \\
Not done & 5 & 16.7 \\
BRCA1 & 0 & 0 \\
BRCA2 & 1 & 3.3 \\
NF1 & 1 & 3.3 \\
Family history of breast cancer & 19 & 63.3 \\
Prior breast cancer & 4 & 13.3 \\
Prior breast surgery & 9 & 30.0 \\
Right lumpectomy & 3 & 10.0 \\
Left lumpectomy & 1 & 3.3 \\
Reduction & 2 & 6.6 \\
Augmentation & 4 & 13.3 \\
Other & 4 & 3.3 \\
Prior radiation therapy & 3 & 30.0 \\
Post-mastectomy radiation therapy & 8 & \\
Total radiation (per breast) & 11.0 \\
Neoadjuvant chemotherapy & & \\
Adjuvant chemotherapy & & \\
\hline
\end{tabular}

used vicryl mesh only. More unilateral cases were performed during R-C19 $(p<0.001)$. Fewer nipple-sparing mastectomies were performed during R-C19 $(p=0.0387)$. There was no difference in reconstruction type (implant versus expander) between pre-C19 and R-C19 $(p=0.1333)$. There was a trend toward more prepectoral reconstruction during R-C19 (0.0669) (Table 1).

Complications during $\mathrm{R}-\mathrm{C} 19$ requiring reoperation occurred in 2 breasts $(5.9 \%)$. Three patients (3 breasts-8.8\%) experienced minor complications. There were no venous thromboembolic events or mortalities during $\mathrm{R}-\mathrm{C} 19$. Comparison of pre-C19 and R-C19 revealed no difference in operative complication rate $(p=0.6419)$ or minor complication rate $(p=0.6617)$ (Table 2$)$.

Length of stay during R-C19 was 0 nights (same-day discharge) in 28 patients (93.3\%) and 1 night in 2 patients (6.7\%). Enhanced recovery after surgery protocol (ERAS) specific for mastectomy with immediate reconstruction was utilized during R-C19 pre-operatively in 24 patients (85.7\%), and $100 \%$ of patients received pre-operative paravertebral blocks. No breast surgeons or plastic surgeons (including housestaff) tested positive for COVID-19 or had symptoms requiring missing days of work as a result of these operations.

\section{Discussion}

This study shows that despite surging cases of COVID-19, patients were able to undergo immediate prosthetic breast reconstruction in a safe manner, without an increase in complications or adverse events compared with the months prior to the pandemic. Additionally, surgeons and hospital staff providing care to this population of patients were also kept safe during that time. The safety of patients as well as the safety of physicians and healthcare staff who are providing care is paramount. We now understand through experience and the advice of our public health and infectious disease colleagues that COVID-19 will be part of our lives for an extended period of time. In Massachusetts, we overcame a large surge of cases during the months of March and April of 2020. We learned and adapted from our experiences, and now have protocols in place that protect patients, physicians, and hospital staff.

We had begun same-day mastectomy with immediate implant or expander reconstruction approximately 1 month prior to R-C19 [6]. Other institutions also initiated similar protocols during the pandemic [7]. The pandemic restrictions were a catalyst to permit more patients to be discharged same day whenever it was deemed medically appropriate. Patients were educated about the plan for potential same-day discharge prior to surgery, which aided significantly in this process. Over $90 \%$ of our patients were discharged on the same day of mastectomy-reconstruction during R-C19, and we had no returns to the emergency room or readmissions for post-operative pain control or drain concerns. Virtual care platforms were utilized for post-operative visits where appropriate. Drains were removed by visiting nurse assistants at the patient's home when they met removal criteria to avoid patients coming to the clinic whenever possible.

At the present time, our hospital organization (Mass General Brigham-MGB) requires all faculty, staff, and residents to electronically attest daily regarding potential symptoms of COVID-19. During the surge, visitors were not permitted to attend clinic visits or accompany patients undergoing ambulatory surgery. During R-C19, no visitors were permitted for inpatients. There were exceptions to the visitor policy for compassionate care, for births, for pediatric patients, and for patients with disabilities. All patients and visitors must attest regarding potential symptoms prior to 
entering any of our facilities, and they must don a hospital-issued mask and perform hand hygiene upon entering a building.

As of June 1, 2020, MGB started performing COVID-19 PCR testing 48 to $72 \mathrm{~h}$ prior to all elective operations and procedures. This practice has continued regardless of vaccination status. If patients test negative, standard precautions are taken in the operating room, including the use of level 2 surgical masks and eye protection. Enhanced respiratory isolation protocols including the use of N95 masks are used only in positive COVID-19 cases or in cases where the patient's COVID-19 status is unknown.

As we move forward within this "new normal", we are learning about the chronic effects of COVID-19 on patients who have survived acute infection. There are now studies that are aiding to determine the appropriate timing of elective surgery in patients who are COVID-recovered $[8,9]$.

This study provides evidence that immediate prosthetic breast reconstruction is feasible and safe during the COVID19 pandemic. The complication rate during the first surge of COVID-19 was not different than prior to the pandemic, which indicates it may not be necessary in future surges to postpone bilateral operations, provided that the appropriate resources are available to care for this cohort of patients.

\section{Funding None.}

Availability of data and materials The data that support the findings of this study are available from Dr. Faulkner but restrictions apply to the availability of these data, which were used under licence for the current study, and so are not publicly available. Data are, however, available from the authors upon reasonable request and with permission of the Mass General Brigham IRB.

Code availability Not applicable.

\section{Declarations}

Conflicts of interest None.

Ethics approval Not applicable.

Consent to participate Not applicable.

Consent for publication All authors consent to publication of this article.

\section{References}

1. Massachusetts COVID-19 State of Emergency. https://www.mass. gov/info-details/covid-19-state-of-emergency. Accessed $27 \mathrm{Sept}$ 2020.

2. Dietz JR, et al. Recommendations for prioritization, treatment, and triage of breast cancer patients during the COVID-19 pandemic. the COVID-19 pandemic breast cancer consortium. Breast Cancer Res Treat. 2020;181(3):487-97. https://doi.org/10.1007/ s10549-020-05644-z.

3. Smith BL, et al. A system for risk stratification and prioritization of breast cancer surgeries delayed by the COVID-19 pandemic: preparing for re-entry. Breast Cancer Res Treat. 2020;183(3):51524. https://doi.org/10.1007/s10549-020-05792-2.

4. ASPS Statement on Breast Reconstruction in the face of COVID19 Pandemic. https://www.plasticsurgery.org/documents/medic al-professionals/COVID19-Breast-Reconstruction-Statement.pdf. Accessed 27 Sept 2020.

5. Evans DGR, et al. Breast cancer in neurofibromatosis 1: survival and risk of contralateral breast cancer in a five country cohort study. Genet Med Off J Am Coll Med Genet. 2020;22(2):398-406. https://doi.org/10.1038/s41436-019-0651-6.

6. Specht M, et al. High-efficiency same-day approach to breast reconstruction during the COVID-19 crisis. Breast Cancer Res Treat. 2020;182(3):679-88. https://doi.org/10.1007/ s10549-020-05739-7.

7. Perez-Alvarez IM, et al. Breast surgery in the time of global pandemic: benefits of same-day surgery for breast cancer patients undergoing mastectomy with immediate reconstruction during COVID-19. Plast Reconstr Surg. 2020;146(4):522e-3e. https:// doi.org/10.1097/PRS.0000000000007269.

8. Thyagarajan R, Mondy K. Timing of surgery after recovery from coronavirus disease 2019 (COVID-19) infection. Infect Control Hosp Epidemiol. 2021;42(6):790-1. https://doi.org/10.1017/ice. 2020.325.

9. El-Boghdadly K, et al. SARS-CoV-2 infection, COVID-19 and timing of elective surgery: a multidisciplinary consensus statement on behalf of the Association of Anaesthetists, the Centre for Peri-operative Care, the Federation of Surgical Specialty Associations, the Royal College of Anaesthetists and the Royal College of Surgeons of England. Anaesthesia. 2021;76(7):940-6. https:// doi.org/10.1111/anae.15464.

Publisher's Note Springer Nature remains neutral with regard to jurisdictional claims in published maps and institutional affiliations. 